Supporting Information for

\title{
Reducing heat duty of MEA regeneration using sulfonic acid functionalized mesoporous MCM-41 catalyst
}

Qiang Sun ${ }^{\mathrm{a}}$, Tianhao Li ${ }^{\mathrm{a}}$, Yu Mao ${ }^{\mathrm{a}}$, Hongxia Gao ${ }^{\mathrm{a}}$ *, Teerawat Sema ${ }^{\mathrm{b}}$, Shengyu Wang ${ }^{\mathrm{a}}$, Libin

$$
\text { Liua, Zhiwu Liang a, * }
$$

aJoint International Center for $\mathrm{CO}_{2}$ Capture and Storage (iCCS), Provincial Hunan Key Laboratory for Cost-effective Utilization of Fossil Fuel Aimed at Reducing $\mathrm{CO}_{2}$ Emissions, College of Chemistry and Chemical Engineering, Hunan University, Changsha, 410082, PR China

${ }^{b}$ Department of Chemical Technology, Faculty of Science, Chulalongkorn University, Bangkok, 10330, Thailand

* Author for correspondence:

Email: hxgao@hnu.edu.cn (Dr. H Gao), zwliang@hnu.edu.cn (Dr. Z Liang) 


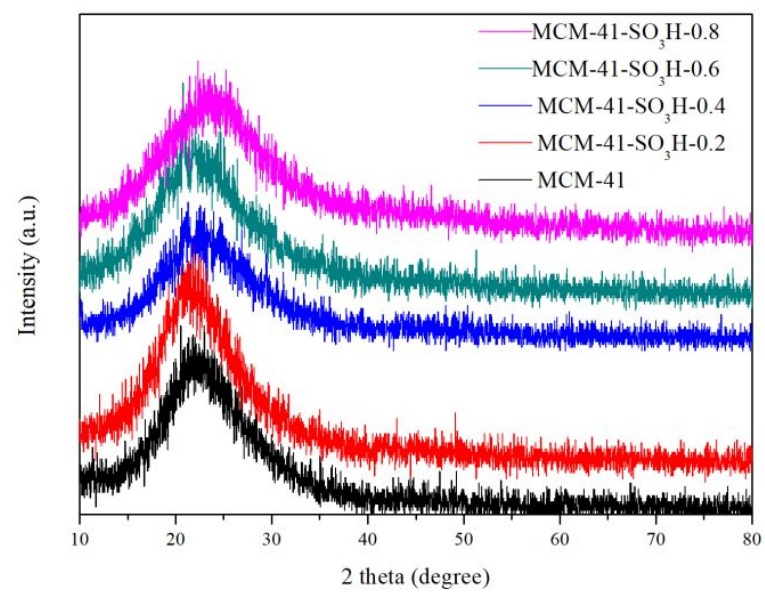

Figure S1. XRD curves.

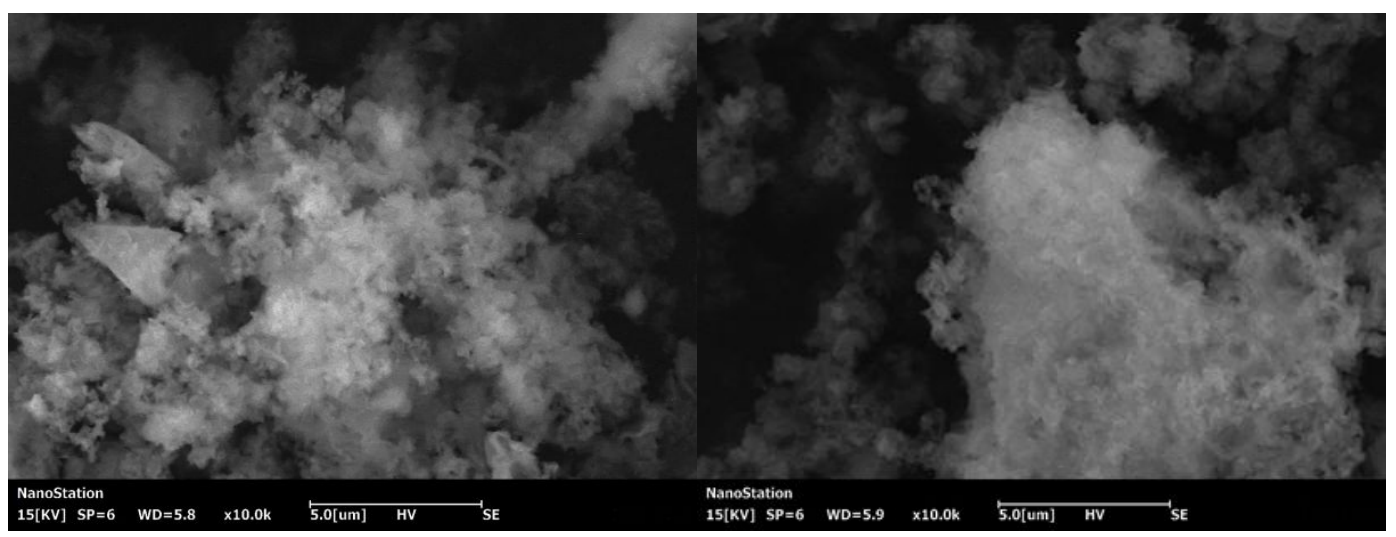

(a)

(b)

Figure S2. SEM image of (a) MCM-41, (b) MCM-41-SO ${ }_{3} \mathrm{H}-0.6$. 

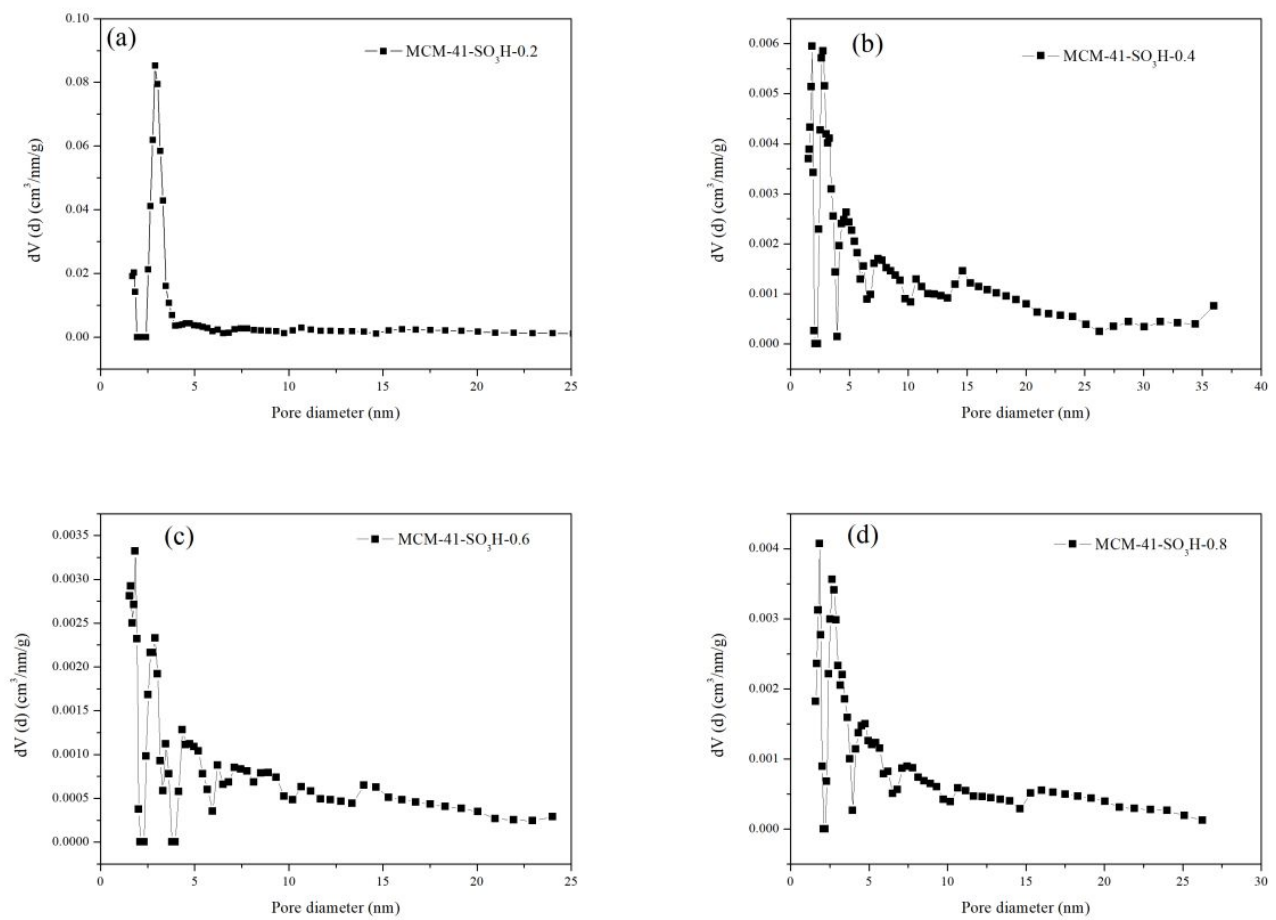

Figure S3. Pore size distributions of the $\mathrm{MCM}-41-\mathrm{SO}_{3} \mathrm{H}$ catalysts.

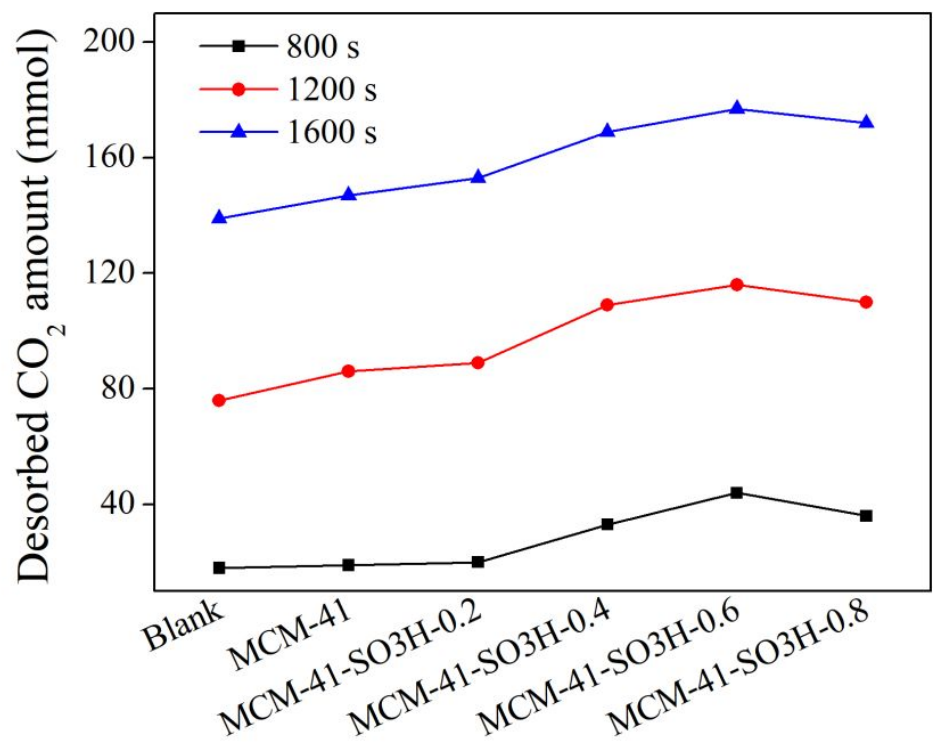

Figure S4. Comparison of cyclic capacity for the different catalysts-MEA systems at different regeneration time. 
Table S1 A brief review of studies for catalyzing $\mathrm{CO}_{2}$ desorption process with different catalysts in the rich $5 \mathrm{M}$ MEA solvent.

\begin{tabular}{|c|c|c|c|}
\hline Catalyst & $\begin{array}{l}\text { Desorption } \\
\text { temperature } \\
\left({ }^{\circ} \mathrm{C}\right)\end{array}$ & Main results & References \\
\hline $\begin{array}{l}\gamma-\mathrm{Al}_{2} \mathrm{O}_{3} / \mathrm{HZSM}- \\
5, \mathrm{HZSM}-5, \mathrm{HY} \\
\gamma-\mathrm{Al}_{2} \mathrm{O}_{3}\end{array}$ & $50-105$ & $\begin{array}{l}\text { Mixture catalysts presented better } \\
\text { performance than single catalysts; } \\
\text { Amount of desorbed } \mathrm{CO}_{2} \text { increased by } \\
20.1-31.2 \% \text {; } \\
\text { Heat duty reduced by } 16.9-23.7 \% \text {. }\end{array}$ & $\begin{array}{l}\text { (Liang et } \\
\text { al., 2016) }\end{array}$ \\
\hline 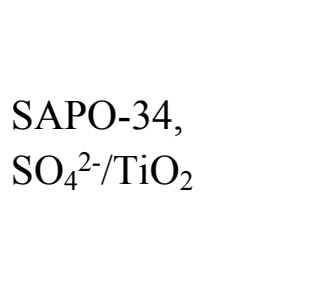 & $70-96$ & $\begin{array}{l}\mathrm{SAPO}-34 \text { showed better performance } \\
\text { than } \mathrm{SO}_{4}^{2-} / \mathrm{TiO}_{2} \text {; } \\
\text { Amount of desorbed } \mathrm{CO}_{2} \text { increased by } \\
14.1-28.2 \% \text {; } \\
\text { Heat duty reduced by } 17.1-24.3 \% \text {. }\end{array}$ & $\begin{array}{l}\text { (Zhang et } \\
\text { al., 2017) }\end{array}$ \\
\hline $\begin{array}{l}\mathrm{HZSM}-5 \\
\mathrm{MCM}-41 \\
\mathrm{SO}_{4}{ }^{2-} / \mathrm{ZrO}_{2}\end{array}$ & $70-98$ & $\begin{array}{l}\text { Performance, HZSM-5 }>\text { MCM- } 41> \\
\mathrm{SO}_{4}{ }^{2-} / \mathrm{ZrO}_{2} \text {; } \\
\text { Amount of desorbed } \mathrm{CO}_{2} \text { increased by } \\
10.6-29.4 \% \text {; } \\
\text { Heat duty reduced by } 9.8-24.8 \% \text {. }\end{array}$ & $\begin{array}{l}\text { (Liu et al., } \\
\text { 2017) }\end{array}$ \\
\hline $\begin{array}{l}\text { Nanoparticles } \\
\mathrm{SiO}_{2}, \mathrm{TiO}_{2} \\
\mathrm{Al}_{2} \mathrm{O}_{3}\end{array}$ & 103 & $\begin{array}{l}\mathrm{The}^{\mathrm{TiO}_{2}} \text { shows best performance, and } \\
\text { the use of } \mathrm{TiO}_{2} \text { nanoparticles saved } \\
\text { desorption time by } 42 \% \text {. } \\
\text { Performance, } \mathrm{MoO}_{3}>\mathrm{V}_{2} \mathrm{O}_{5}>\mathrm{Cr}_{2} \mathrm{O}_{3}>\end{array}$ & $\begin{array}{l}\text { (Wang et } \\
\text { al., 2016) }\end{array}$ \\
\hline $\begin{array}{l}\mathrm{V}_{2} \mathrm{O}_{5}, \mathrm{MoO}_{3}, \\
\mathrm{WO}_{3}, \mathrm{TiO}_{2} \text {, and } \\
\mathrm{Cr}_{2} \mathrm{O}_{3},\end{array}$ & $35-86$ & $\begin{array}{l}\mathrm{TiO}_{2}>\mathrm{WO}_{3} ; \\
\text { Amount of desorbed } \mathrm{CO}_{2} \text { increased by } \\
44-94 \% \text {; } \\
\text { Sensible heat reduced by } 25-48 \% \text {. }\end{array}$ & $\begin{array}{l}\text { (Bhatti et } \\
\text { al., 2017) }\end{array}$ \\
\hline SZMF & $60-98$ & $\begin{array}{l}\text { Amount of desorbed } \mathrm{CO}_{2} \text { increased by } \\
38.1-54.7 \% \text {; } \\
\text { Heat duty reduced by } 27.7-39.4 \% \text {. }\end{array}$ & $\begin{array}{l}\text { (Zhang et } \\
\text { al., 2019) }\end{array}$ \\
\hline $\begin{array}{l}\mathrm{SO}_{4}{ }^{2-} / \mathrm{ZrO}_{2-} \\
\mathrm{HZSM}-5\end{array}$ & 98 & $\begin{array}{l}\text { Amount and rate of } \mathrm{CO}_{2} \text { desorption } \\
\text { increased by } 40 \text { and } 37 \% \text {; } \\
\text { Energy consumption reduced by } \\
\text { approximately } 31 \% \text {. }\end{array}$ & $\begin{array}{l}\text { (Xing et } \\
\text { al., 2020) }\end{array}$ \\
\hline $\begin{array}{l}\mathrm{SO}_{4}^{2-} / \mathrm{ZIF}-67- \\
\mathrm{C} @ \mathrm{TiO}_{2}\end{array}$ & 88 & $\begin{array}{l}\text { Amount and rate of } \mathrm{CO}_{2} \text { desorption } \\
\text { increased by } 64.5 \text { and } 153 \% \text {; } \\
\text { Energy consumption reduced by } \\
\text { approximately } 36 \% \text {. }\end{array}$ & $\begin{array}{l}\text { (Xing et } \\
\text { al., 2021) }\end{array}$ \\
\hline
\end{tabular}


Table S2 Nomenclature.

\begin{tabular}{|c|c|c|c|}
\hline \multicolumn{4}{|c|}{ Nomenclature } \\
\hline \multicolumn{4}{|c|}{ Abbreviations } \\
\hline $\mathrm{CO}_{2}$ & carbon dioxide & $\mathrm{TG}$ & Thermo-gravimetric analysis \\
\hline $\mathrm{CCS}$ & $\mathrm{CO}_{2}$ capture and storage & $\mathrm{BET}$ & Brunauer-Emmett-Teller \\
\hline MEA & monoethanolamine & $\mathrm{BJH}$ & Barrett-Joyner-Halenda \\
\hline XRD & X-ray diffraction & MSA & $\begin{array}{l}\text { mesoporous surface area } \\
\left(\mathrm{m}^{2} / \mathrm{g}\right)\end{array}$ \\
\hline SEM & Scanning electron microscopy & TAS & Total acid sites (mmol/g) \\
\hline FT-IR & $\begin{array}{l}\text { Fourier transform infrared } \\
\text { spectrometer }\end{array}$ & & \\
\hline Symbo & & & \\
\hline$r$ & $\begin{array}{l}\mathrm{CO}_{2} \text { desorption rate } \\
(\mathrm{mol} /(\mathrm{s} * \mathrm{~L}))\end{array}$ & $X_{\mathrm{CO}_{2}}^{\text {out }}$ & $\begin{array}{l}\text { volumetric fraction of } \mathrm{CO}_{2} \text { in } \\
\text { outlet gas }\end{array}$ \\
\hline$V$ & $\begin{array}{l}\text { volume of the desorbed MEA } \\
\text { solution (L) }\end{array}$ & HD & $\begin{array}{l}\text { regeneration heat duty } \\
(\mathrm{kJ} / \mathrm{mol})\end{array}$ \\
\hline$v_{N_{2}}^{i n}$ & flow rate of $\mathrm{N}_{2}(\mathrm{~L} / \mathrm{min})$ & $\mathrm{RH}$ & relative heat duty $(\%)$ \\
\hline
\end{tabular}


Table S3. Mass balance of the gas phase and liquid methods after $1 \mathrm{~h}$ for $\mathrm{CO}_{2}$ desorption at $90{ }^{\circ} \mathrm{C}$

\begin{tabular}{|c|c|c|c|}
\hline Catalyst system & $\begin{array}{l}\text { Gas method (mol } \\
\mathrm{CO}_{2} / \text { mol MEA) }\end{array}$ & $\begin{array}{l}\text { Liquid method (mol } \\
\left.\mathrm{CO}_{2} / \mathrm{mol} \mathrm{MEA}\right)\end{array}$ & $\operatorname{ARD}(\%)$ \\
\hline Blank & 0.4346 & 0.4376 & 0.69 \\
\hline MCM-41 & 0.4333 & 0.4395 & 1.41 \\
\hline $\mathrm{SO}_{4}{ }^{2-} / \mathrm{MCM}-41$ & 0.4314 & 0.4409 & 2.15 \\
\hline $\mathrm{MCM}-41-\mathrm{SO}_{3} \mathrm{H}-0.2$ & 0.4287 & 0.4384 & 2.21 \\
\hline $\mathrm{MCM}-41-\mathrm{SO}_{3} \mathrm{H}-0.4$ & 0.4270 & 0.4353 & 1.91 \\
\hline $\mathrm{MCM}-41-\mathrm{SO}_{3} \mathrm{H}-0.6$ & 0.4280 & 0.4394 & 2.60 \\
\hline MCM-41-SO ${ }_{3} \mathrm{H}-0.8$ & 0.4253 & 0.4389 & 3.09 \\
\hline MCM-41-SO ${ }_{3} \mathrm{H}-0.6-1^{\text {cycle }}$ & 0.4303 & 0.4471 & 3.76 \\
\hline MCM-41-SO ${ }_{3} \mathrm{H}-0.6-2^{\text {cycle }}$ & 0.4324 & 0.4356 & 0.73 \\
\hline MCM-41-SO ${ }_{3} \mathrm{H}-0.6-3^{\text {cycle }}$ & 0.4289 & 0.4387 & 2.23 \\
\hline MCM-41-SO ${ }_{3} \mathrm{H}-0.6-4^{\text {cycle }}$ & 0.4277 & 0.4399 & 2.77 \\
\hline MCM-41-SO 3 H-0.6-5 cycle & 0.4325 & 0.4428 & 2.33 \\
\hline
\end{tabular}

Table S4. The effect of MCM-41-SO $\mathrm{S}_{3} \mathrm{H}$ catalysts on the desorption performance at $90{ }^{\circ} \mathrm{C}$

\begin{tabular}{ccc}
\hline Catalysts & $\begin{array}{c}\text { MSA } \times \mathrm{TAS} \\
\left(\mathrm{m}^{2 *} \mathrm{mmol} / \mathrm{g}^{2}\right)\end{array}$ & $\mathrm{HD}(\mathrm{kJ} / \mathrm{mol})$ \\
\hline Blank & - & 2880.00 \\
MCM-41-SO $3 \mathrm{H}-0.2$ & 48.30 & 2522.03 \\
MCM-41-SO ${ }_{3} \mathrm{H}-0.4$ & 54.90 & 2052.41 \\
MCM-41-SO & & 1920.00 \\
MCM-0.6 & 66.02 & 2038.36 \\
\hline
\end{tabular}


Bhatti, U.H., Shah, A.K., Kim, J.N., You, J.K., Choi, S.H., Lim, D.H., Nam, S., Park, Y.H., Baek, I.H., 2017. Effects of transition metal oxide catalysts on MEA solvent regeneration for the post-combustion carbon capture process. Acs Sustainable Chemistry \& Engineering 5, $5862-5868$.

Liang, Z., Idem, R., Tontiwachwuthikul, P., Yu, F., Liu, H., Rongwong, W., 2016. Experimental study on the solvent regeneration of a $\mathrm{CO}_{2}$-loaded MEA solution using single and hybrid solid acid catalysts. AIChE Journal 62, 753-765.

Liu, H., Zhang, X., Gao, H., Liang, Z., Idem, R., Tontiwachwuthikul, P., 2017. Investigation of $\mathrm{CO}_{2}$ regeneration in single and blended amine solvents with and without catalyst. Industrial \& Engineering Chemistry Research 56, 7656-7664.

Wang, T., Yu, W., Liu, F., Fang, M., Farooq, M., Luo, Z., 2016. Enhanced $\mathrm{CO}_{2}$ absorption and desorption by monoethanolamine (MEA)-based nanoparticle suspensions. Industrial \& Engineering Chemistry Research 55, 7830-7838.

Xing, L., Wei, K., Li, Y., Fang, Z., Li, Q., Qi, T., An, S., Zhang, S., Wang, L., 2021. TiO 2 Coating Strategy for Robust Catalysis of the Metal-Organic Framework toward EnergyEfficient $\mathrm{CO}_{2}$ Capture. Environmental Science \& Technology 55, 11216-11224.

Xing, L., Wei, K.X., Li, Q.W., Wang, R.J., Zhang, S.H., Wang, L.D., 2020. One-Step Synthesized $\mathrm{SO}_{4}{ }^{2-} / \mathrm{ZrO}_{2}-\mathrm{HZSM}-5$ Solid Acid Catalyst for Carbamate Decomposition in $\mathrm{CO}_{2}$ Capture. Environmental Science \& Technology 54, 13944-13952. 
Zhang, X., Zhang, X., Liu, H., Li, W., Xiao, M., Gao, H., Liang, Z., 2017. Reduction of energy requirement of $\mathrm{CO}_{2}$ desorption from a rich $\mathrm{CO}_{2}$-loaded $\mathrm{MEA}$ solution by using solid acid catalysts. Applied Energy 202, 673-684.

Zhang, X., Zhu, Z., Sun, X., Yang, J., Gao, H., Huang, Y., Luo, X., Liang, Z., Tontiwachwuthikul, P., 2019. Reducing Energy Penalty of $\mathrm{CO}_{2}$ Capture Using Fe Promoted $\mathrm{SO}_{4}{ }^{2-} / \mathrm{ZrO}_{2} / \mathrm{MCM}-41$ Catalyst. Environmental Science \& Technology 53, 6094-6102. 